\title{
Influence de l'âge des moutons et de l'ajout de méthionine hydroxy analogue sur l'efficacité de mastication
}

\author{
E Amouche, AG Deswysen \\ Université catholique Louvain, Fac Sci Agr, unité de Génétique, place Croix-du-Sud, \\ 2 (bte 14), B-1348, Louvain-la-Neuve, Belgique
}

L'activité masticatoire des ruminants joue un rôle majeur dans la réduction de la taille et l'augmentation de la densité spécifique des particules alimentaires. L'objectif de ce travail est d'étudier l'influence de l'âge des ovins et de l'ajout de méthionine hydroxy analogue sur l'indice et l'efficacité de mastication.

Douze moutons mâles castrés, 6 âgés de 6 mois $(32,5 \mathrm{~kg})$ et 6 âgés de 30 mois $(83 \mathrm{~kg})$, de race Texel, reçurent selon un schéma expérimental en crossover $2 \times 2 \times 6(2 \times 21$ j) pour chaque âge, 2 types de rations composées d'ensilage d'herbe préfanée ad libitum et de $100 \mathrm{~g} / \mathrm{i}$ de tourteau de soja contenant 0 ou $5 \mathrm{~g}$ de méthionine hydroxy analogue sous une forme acide (MHA). Ils furent logés dans des cages à métabolisme. Les mouvements de mâchoires furent enregistrés en continu durant $4 \mathrm{j}$ (12 au $15^{\mathrm{e}}$ j) et la collecte totale des matières fécales fut réalisée de manière concomitante. La distribution moyenne de la taille des particules fécales
(DMPF) fut déterminée par tamisage sous eau. L'efficacité de mastication (EM; $g$ MS fèces $x$ $\mathrm{mm}$ (DMPaliment - DMPF)/min) fut calculée de manière semblable à Deswysen et Ellis (1990).

Seul chez les moutons âgés de 6 mois, l'apport de MHA a significativement réduit l'ingestion volontaire (IV) et augmenté l'indice de mastication (IM; $\mathrm{min} / \mathrm{kg} \mathrm{MS}$ ), entraînant donc une réduction de la DMPF et de l'EM. L'IV et I'IM furent plus élevés chez les jeunes moutons comparés aux adultes, alors que l'EM fut plus faible. La DMPF ne présenta toutefois aucune différence notable entre les 2 âges, suggérant que la DMP digesta n'est pas le seul facteur déterminant du transit par l'orifice réticuloomasal.

Deswysen AG, Ellis WC (1990) J Anim Sci 68, $3871-3879$

Tableau I. Ingestion volontaire (IV), indice de mastication (IM), distribution moyenne de la taille des particules fécales (DMPF) et efficacité de mastication (EM) chez des moutons âgés de 6 et 30 mois.

\begin{tabular}{|c|c|c|c|c|c|c|c|c|c|}
\hline \multirow[t]{2}{*}{ Paramètres } & \multicolumn{4}{|c|}{ Moutons âgés de 6 mois } & \multicolumn{4}{|c|}{ Moutons âgés de 30 mois } & \multirow{2}{*}{$\begin{array}{c}\text { Valeur } P \\
\text { Áge }\end{array}$} \\
\hline & Témoin & MHA & $E S$ & $P$ & Témoin & $M H A$ & $E S$ & $P$ & \\
\hline IV (g MS/kg Po,75/j) & 54,4 & 52,4 & 0,3 & 0,01 & 38,8 & 39,8 & 0,3 & 0,11 & $<0,001$ \\
\hline $\mathrm{IM}(\mathrm{min} / \mathrm{kg} \mathrm{MS})$ & 1071,4 & 1140,0 & 19,5 & 0,07 & 679,2 & 674,4 & 25,4 & 0,90 & $<0,001$ \\
\hline DMPF $(\mu \mathrm{m})$ & 180,1 & 161,3 & 4,0 & 0,03 & 167,2 & 164,1 & 3,8 & 0,59 & 0,25 \\
\hline $\mathrm{EM}(\mathrm{g} \mathrm{MS} \cdot \mathrm{mm} / \mathrm{min})$ & 6,7 & 5,9 & 0,1 & 0,01 & 10,9 & 11,4 & 0,5 & 0,56 & $<0,001$ \\
\hline
\end{tabular}

